\title{
1 Risks in signal processing pipelines influencing the estimation of phase dependency for EEG-TMS
}

4 Robert Guggenberger ${ }^{*}$, Maximilian Scherer, Alireza Gharabaghi*

5 Division of Functional and Restorative Neurosurgery, and Tuebingen Neuro Campus,

6 Eberhard Karls University Tuebingen, Tuebingen, Germany

7 *To whom correspondence should be addressed

8 Dr. Robert Guggenberger and Professor Alireza Gharabaghi, Division of Functional

9 and Restorative Neurosurgery, Eberhard Karls University Tuebingen, Otfried-Mueller-

10 Str.45, 72076 Tuebingen, Germany. Email addresses: robert.guggenberger@uni-

11 tuebingen.de and alireza.gharabaghi@uni-tuebingen.de

12

\section{Acknowledgments}

14 M.S. was supported by the Graduate Training Centre of Neuroscience \& 15 International Max Planck Research School, Graduate School of Neural Information 16 Processing, Tuebingen, Germany. A.G. was supported by grants from the German 17 Federal Ministry of Education and Research [BMBF 13GW0119B, IMONAS; 18 13GW0214B, INSPIRATION; 13GW0270B, INAUDITAS] and the Baden19 Wuerttemberg Foundation [NEU005, NemoPlast]. The authors declare no competing 20 financial interests. 


\section{Abstract}

23 Phase-dependency of cortico-spinal excitability can be researched using TMS-EEG.

24 Due to the large artifact, non-causal filters can smear the TMS artifact and distort the

25 phase. However, causal filters can become biased by too high filter orders or uneven

26 pass-bands. We explored the influence of different signal processing pipelines on the

27 estimation of the optimal phase. This exploration involved performing two simulation

28 studies. In the first, we simulated two different phase-dependencies (uni- versus

29 bimodal) and sought to recover them with two distinct approaches that have previously

30 been described. In the second, we specifically explored how filter parameters (e.g.,

31 order, pass-band) biased the phase estimation. On the basis of these findings, we

32 propose using up-to-date toolboxes, re-running scripts after software updates and

33 performing simulation studies in parallel to safeguard the analysis pipeline of empirical

34 studies.

35 Keywords: phase dependency, transcranial magnetic stimulation, motor-evoked

36 potential, digital signal processing, brain-state-dependent stimulation 


\section{Introduction}

38 The excitability of neuronal populations depends on their current state (Izhikevich,

39 2007). The use of non-invasive measurements to monitor the current state of neuronal

40 populations is an important step towards implementing brain-state-dependent

41 transcranial stimulation for research and therapy. This is feasible with power-based

42 approaches. For example, desynchronization of sensorimotor power, as picked up by

43 electroencephalography (EEG), increases cortico-spinal excitability (CSE), as

44 measured by the motor-evoked potential (MEP) in response to transcranial magnetic

45 stimulation (TMS; Takahashi et al., 2018; Takemi et al., 2013; Kraus et al., 2016,

46 2018). Similarly, transcranial alternating current stimulation (tACS), which is assumed

47 to synchronize neuronal populations (Zaehle et al., 2010), is able to increase CSE.

48 Such knowledge has already been used to implement interventions for clinical

49 application (Gharabaghi et al., 2014).

50 Most TMS devices that apply short-lasting single pulses can be triggered with very

51 short latencies. Such high temporal precision makes it possible to probe the state-

52 dependency of CSE not only with respect to the rather slow-changing measure of

53 oscillatory power but also with regard to the relatively fast-changing phase of an

54 oscillation. Research has suggested that the use of TMS at different phases of

55 sinusoidal signals applied via transcranial alternating current stimulation (tACS)

56 discloses such phase-dependency of CSE (Raco et al., 2016; Guerra et al., 2016;

57 Nakazono et al., 2016; Fehér et al., 2017; Schilberg et al., 2018).

58 The step from exogenous entrainment of oscillations via tACS to reading the current

59 endogenous phase of an oscillation from EEG or EMG recordings entails several

60 challenges. Unlike a tACS signal, the signal-to-noise ratio of non-invasive

61 electrophysiological recordings is low. The amplitude and phase of oscillations are

62 non-stationary and might be sinusoidal only in approximation (Cole and Voytek, 2017).

63 In an attempt to address this non-stationarity, the construction of dedicated hardware

64 might become necessary for fast processing. However, despite using dedicated

65 hardware, and even if performed only when the oscillation amplitudes are high, real-

66 time estimation of an oscillations phase might have a standard deviation of about $50^{\circ}$

67 (Zrenner et al., 2017).

68 Post-hoc analysis of TMS, applied initially at random and subsequently probed for

69 possible phase-dependencies, therefore remains an important method of research

70 (van Elswijk et al., 2010; Keil et al., 2013; Khademi et al., 2018). Nonetheless, this

71 approach still requires the stringent design of a signal processing pipeline with a

72 special focus on the filter design. For example, non-causal filters may smear the TMS

73 artifact and distort the phase of an oscillation prior to the TMS pulse. Furthermore,

74 when transferred to real-time applications, only causal filters are feasible. In this study,

75 we show how methodological differences in the design of causal filters affect the

76 estimation of the phase dependency. This is not simply a methodological question.

77 For example, with regard to the phase-dependency in the oscillatory beta-band, it is

78 unclear as to whether there is only one maximum of CSE, found in the rising phase of

79 an oscillation (Khademi et al., 2018; van Elswijk et al., 2010), or whether there are two

80 maxima of CSE, i.e., at the peak and trough of an oscillation (Keil et al., 2013). Using

81 simulated data, we show how two different approaches of phase estimation can result

82 in such conflicting findings, even when based on identical data. Subsequently, we

83 explore the general influence of the filter order and bandwidth on recovering the phase 
84 of maximal CSE. Finally, we discuss policies for minimizing risks in the design and

85 implementation of signal processing pipelines for estimation of phase dependency.

\section{Material and Method}

87 All simulations were performed and visualized with Anaconda Python 3.6.5 on Linux

88 Mint 18.2, employing SciPy 1.1.0, NumPy 1.14.3, Seaborn 0.8.1 and Matplotlib 2.2.2.

89 The script to create simulated dependencies is available online (https://osf.io/mgu4h/).

\section{Simulation 1}

91 We simulated data with two dependencies between oscillatory phase and MEP

92 amplitude. One was bimodal, i.e., with two maxima of MEP at peak and trough of the

93 oscillation. The other was unimodal, i.e., with only a single maximum at the rising flank.

94 Both dependencies were sampled, filtered and processed in accordance with the

95 methods reported previously (Keil et al., 2013; van Elswijk et al., 2010).

\section{Data model}

97 In the dependency models, data was simulated as a $18 \mathrm{~Hz}$ cosine signal, and

98 subsequent analyses were performed in accordance with two previous reports. The

99 first approach involved estimating the phase in analogy to Keil and colleagues (Keil et

100 al., 2013) on the basis of a Hilbert transformation. We simulated the data with a $2 \mathrm{kHz}$

101 sampling rate and duration of $\pm 1.5 \mathrm{~s}$, and included three cycles, terminating $5 \mathrm{~ms}$

102 before the TMS pulse. The second approach entailed estimating the phase in analogy

103 to Elswijk and colleagues (van Elswijk et al., 2010) on the basis of a discrete Fourier

104 transformation. We simulated the data with a $10 \mathrm{kHz}$ sampling rate and duration of \pm

$1051.1 \mathrm{~s}$, and included two cycles prior to the TMS pulse.

\section{MEP dependency}

107 We simulated two different dependencies between the oscillatory phase and MEP

108 amplitude: maximum MEP amplitude (i) with a unimodal pattern, i.e., at the rising

109 phase of $18 \mathrm{~Hz}$ oscillations, (ii) with a bimodal pattern, i.e., at the peak and trough of

$11018 \mathrm{~Hz}$ oscillations. We simulated a log-linear relationship with base 50 to account for

111 the log-normal distribution of MEPs. The two dependency models are presented in

112 figure $1 \mathrm{~A}$.

\section{Filter Order}

114 Simulated data epochs were bandpass filtered forward in time with a Butterworth filter 115 with orders from 0 to 8, and for two different frequency bands depending on the

116 approach, i.e., 10-400 Hz (van Elswijk et al., 2010) or 17-19 Hz (Keil et al., 2013).

\section{Assessment}

118 We visualized the simulation results as polar-linear and linear-linear scatter plots

119 between oscillatory phase and MEP amplitude. To achieve a well-resolved and

120 uniform sampling across the unit circle, the relationship for each integer phase was

121 sampled from $0^{\circ}$ to $360^{\circ}$. For the simulation, we added a small white noise term (with

1220.05 standard deviation) to prevent points from overlaying and to reduce any possible

123 bias due to numerical errors. 


\section{Simulation 2}

125 We simulated data as above, but with one dependency mode only, i.e., peak of CSE

126 would be at the rising flank of an oscillation at $18 \mathrm{~Hz}$. Signals were simulated with a

127 sampling rate of $1000 \mathrm{~Hz}$, for the duration of $\pm 1.5 \mathrm{~s}$ around the TMS for filtering, and

128 included three cycles for subsequent phase estimation. The signal was simulated

129 without noise.

\section{Data processing}

131 We filtered the data by exploring the influence of four parameters, i.e., estimation

132 method, filter order, filter bandwidth, and filter center. The phase was estimated using

133 either Hilbert or Fourier transformation as described above. Additionally, we

134 systematically increased filter order up to an order of 8, and decreased the filter

135 bandwidth. This was achieved in two ways, with the bandwidth (i) centered on the

136 frequency of interest, and (ii) unevenly anchored.

\section{Assessment}

138 We visualized the simulation results as heat maps, showing the phase which was

139 estimated to exhibit a maximal MEP after filtering.

\section{Results}

\section{Simulation study 1}

142 Our first simulation contrasted a narrow-band centered filter of high order followed by

143 Hilbert transformation (approach 1) with a broad-band unevenly anchored filter of

144 lower order followed by Fourier transformation (approach 2). It suggested that the

145 second approach achieves better recovery of the original dependency. The first

146 approach was unable to recover a unimodal phase-dependency of MEP amplitudes,

147 while the second was able to discern between uni- and bimodal phase dependencies

148 (see figure $1 \mathrm{~B} / \mathrm{C}$ ). Only when the order of the narrow-band filter was decreased did

149 this approach recover a unimodal phase dependency (see figure 1 D) and distinguish

150 it from a bimodal phase dependency (see figure $1 \mathrm{E}$ ).

\section{Simulation study 2}

152 This simulation explored how the estimation of the optimal phase in the case of

153 unimodal dependency was affected by various parameters of the signal processing

154 pipeline. It highlighted that the phase, whether estimated with Hilbert (see figure $2 \mathrm{~A} / \mathrm{C}$ )

155 or Fourier transformation (see figure $2 \mathrm{~B} / \mathrm{D}$ ), appears to exhibit similar profiles. The

156 main issue comes with higher filter orders by starting to distort the signal; a

157 phenomenon which appears to start earlier for more narrow bands. At the same time,

158 running a causal filter with an uneven bandwidth can bias the optimal phase estimate

159 significantly (see figure $2 \mathrm{C} / \mathrm{D}$ ).

\section{Discussion}

161 We conducted two simulation studies to explore how differences in signal processing

162 affect the estimation of phase-dependency. The first of these studies applied two

163 approaches based on earlier research (Keil et al., 2013; van Elswijk et al., 2010). The

164 second explored the influence of processing on the phase estimation. The goal was to 
165 investigate how differences of data processing can explain supposedly contradictory

166 findings in earlier literature, and explore the related pitfalls.

\section{Methodological considerations}

168 One key result of our simulation studies was that a low filter order is required to 169 recover uni- and bimodal phase-dependency patterns. If filter orders are too high, the

170 signal becomes distorted, which in turn leads to an artifactual bimodal dependency

171 (see figure $1 \mathrm{D} / \mathrm{E}$ ). Given a sufficiently low order, both broad-band Fourier and narrow-

172 band Hilbert transformation were able to recover the original phase-dependent

173 relationship. The apparently bimodal dependency introduced by filtering is mainly

174 artifactual, given that too high filter orders can corrupt the phase spectrum of a signal

175 (Oppenheim et al., 2014).

176 At the same time, applying causal filters with uneven pass-bands induces phase-

177 shifts. An evenly centered passband should therefore be considered as a step towards

178 minimizing phase distortion, should a specific frequency be of interest.

179 More generally, if Hilbert transformation is used for phase-estimation, this can clash

180 with the goal of estimating the phase for a narrow frequency band of interest. In such

181 cases, phase estimation using Fourier transformation after broad-band low-order

182 filtering might constitute a more suitable approach.

183 It should also be noted that using a temporal lag in relationship to the MEP necessarily 184 introduces a phase-shift (see figure $1 \mathrm{D} / \mathrm{E}$ ). For example, $5 \mathrm{~ms}$ at a frequency of $18 \mathrm{~Hz}$ 185 correspond to $\sim 32^{\circ}$. Correcting for this can be challenging. If the frequency is

186 determined a priori, correcting the bias may be a good alternative. However, if more

187 non-stationarity is required, unsupervised phase prediction, e.g., the use of an

188 adaptive autoregressive model (Zrenner et al., 2017) might also be considered as a

189 feasible option.

\section{Toolbox selection}

191 Implementations of the same processing pipeline in different environments, e.g., by

192 using different toolboxes or programming languages, can influence the results

193 (Widmann et al., 2015). Notably, recent versions of a commonly used data analysis

194 software (Fieldtrip, Oostenveld et al., 2011) support an automatic filter instability

195 correction and interrupt the computation (e.g., "fail with an exception"), if the filter

196 order is too high. This behavior was introduced in early 2013 (according to git log-grep

197 "instability", commit \#914d6ab, https://github.com/fieldtrip/fieldtrip/); analyses

198 performed prior to this software update might therefore not have experienced any error

199 warning if the applied filter orders were too high.

\section{Conclusions}

201 Findings can become biased by too high filter orders or uneven pass-bands. On a

202 more general account, these observations propose two approaches: (i) Using up-to-

203 date toolboxes and re-running scripts after software updates (Kitzes et al., 2018) and

204 (ii) Running simulation studies in parallel to the actual data processing to safeguard

205 the analysis pipeline against potential pitfalls (Haufe, 2015). 


\section{References}

Cole, S.R., Voytek, B., 2017. Brain Oscillations and the Importance of Waveform Shape. Trends Cogn. Sci. 21, 137-149. https://doi.org/10.1016/j.tics.2016.12.008

Fehér, K.D., Nakataki, M., Morishima, Y., 2017. Phase-Dependent Modulation of Signal Transmission in Cortical Networks through tACS-Induced Neural Oscillations. Front. Hum. Neurosci. 11. https://doi.org/10.3389/fnhum.2017.00471

Gharabaghi, A., Kraus, D., Leão, M.T., Spüler, M., Walter, A., Bogdan, M., Rosenstiel, W., Naros, G., Ziemann, U., 2014. Coupling brain-machine interfaces with cortical stimulation for brain-state dependent stimulation: enhancing motor cortex excitability for neurorehabilitation. Front. Hum. Neurosci. 8, 122. https://doi.org/10.3389/fnhum.2014.00122

Guerra A, Pogosyan A, Nowak M, Tan H, Ferreri F, Di Lazzaro V, Brown P (2016) Phase dependency of the human primary motor cortex and cholinergic inhibition cancelation during beta tACS. Cereb Cortex 26:3977-3990.

Haufe, S., 2015. An extendable simulation framework for benchmarking EEG-based brain connectivity estimation methodologies. IEEE, pp. https://doi.org/10.1109/EMBC.2015.7320142

Izhikevich, E.M., 2007. Dynamical systems in neuroscience: the geometry of excitability and bursting, Computational neuroscience. MIT Press, Cambridge, Mass.

Keil, J., Timm, J., Sanmiguel, I., Schulz, H., Obleser, J., Schoenwiesner, M., 2013. Cortical Brain States and Corticospinal Synchronization Influence TMS-evoked motor potentials. J. Neurophysiol. https://doi.org/10.1152/jn.00387.2013

Khademi, F., Royter, V., Gharabaghi, A., 2018. Distinct Beta-band Oscillatory Circuits Underlie Corticospinal Gain Modulation. Cereb. Cortex 28, 1502-1515. https://doi.org/10.1093/cercor/bhy016

Kitzes, J., Turek, D., Deniz, F. (Eds.), 2018. The practice of reproducible research: case studies and lessons from the data-intensive sciences. University of California Press, Oakland, California.

Kraus, D., Naros, G., Bauer, R., Khademi, F., Leão, M.T., Ziemann, U., Gharabaghi, A., 2016. Brain StateDependent Transcranial Magnetic Closed-Loop Stimulation Controlled by Sensorimotor Desynchronization Induces Robust Increase of Corticospinal Excitability. Brain Stimulat. 9, 415-424. https://doi.org/10.1016/j.brs.2016.02.007

Kraus D, Naros G, Guggenberger R, Leão MT, Ziemann U, Gharabaghi A.Recruitment of Additional Corticospinal Pathways in the Human Brain withState-Dependent Paired Associative Stimulation. J Neurosci. 2018 Feb7;38(6):1396-1407. doi: 10.1523/JNEUROSCI.2893-17.2017.

Nakazono H, Ogata K, Kuroda T, Tobimatsu S (2016) Phase and frequency-dependent effects of transcranial alternating current stimulation on motor cortical excitability. PloS One 11:e0162521.

Oostenveld, R., Fries, P., Maris, E., Schoffelen, J.-M., 2011. FieldTrip: Open Source Software for Advanced Analysis of MEG, EEG, and Invasive Electrophysiological Data. Comput. Intell. Neurosci. 2011, 1-9. https://doi.org/10.1155/2011/156869

Oppenheim, A.V., Willsky, A.S., Nawab, S.H., 2014. Signals \& Systems.

Raco, V., Bauer, R., Tharsan, S., Gharabaghi, A., 2016. Combining TMS and tACS for closed-loop phasedependent modulation of corticospinal excitability: A feasibility study. Front. Cell. Neurosci. 10. https://doi.org/10.3389/fncel.2016.00143

Schilberg L, Engelen T, Ten Oever S, Schuhmann T, de Gelder B, de Graaf TA, Sack AT (2018) Phase of beta-frequency tACS over primary motor cortex modulates corticospinal excitability. Cortex 103:142-152.

Takahashi, K., Kato, K., Mizuguchi, N., Ushiba, J., 2018. Precise estimation of human corticospinal excitability associated with the levels of motor imagery-related EEG desynchronization extracted by a locked-in amplifier algorithm. J. NeuroEngineeringRehabil. 15. https://doi.org/10.1186/s12984-018-0440-5 
Takemi, M., Masakado, Y., Liu, M., Ushiba, J., 2013. Event-related desynchronization reflects downregulation of intracortical inhibition in human primary motor cortex 110, 1158-1166. https://doi.org/10.1152/jn.01092.2012

van Elswijk, G., Maij, F., Schoffelen, J.-M., Overeem, S., Stegeman, D.F., Fries, P., 2010. Corticospinal beta-band synchronization entails rhythmic gain modulation. J. Neurosci. Off. J. Soc. Neurosci. 30, 4481-4488. https://doi.org/10.1523/JNEUROSCI.2794-09.2010

Widmann, A., Schröger, E., Maess, B., 2015. Digital filter design for electrophysiological data--a practical approach. J. Neurosci. Methods 250, 34-46. https://doi.org/10.1016/j.jneumeth.2014.08.002

Zaehle, T., Rach, S., Herrmann, C.S., 2010. Transcranial alternating current stimulation enhances individual alpha activity in human EEG. PloSOne 5, e13766. https://doi.org/10.1371/journal.pone.0013766

Zrenner, C., Desideri, D., Belardinelli, P., Ziemann, U., 2017. Real-time EEG-defined excitability states determine efficacy of TMS-induced plasticity in human motor cortex. Brain Stimulat. https://doi.org/10.1016/j.brs.2017.11.016 
A

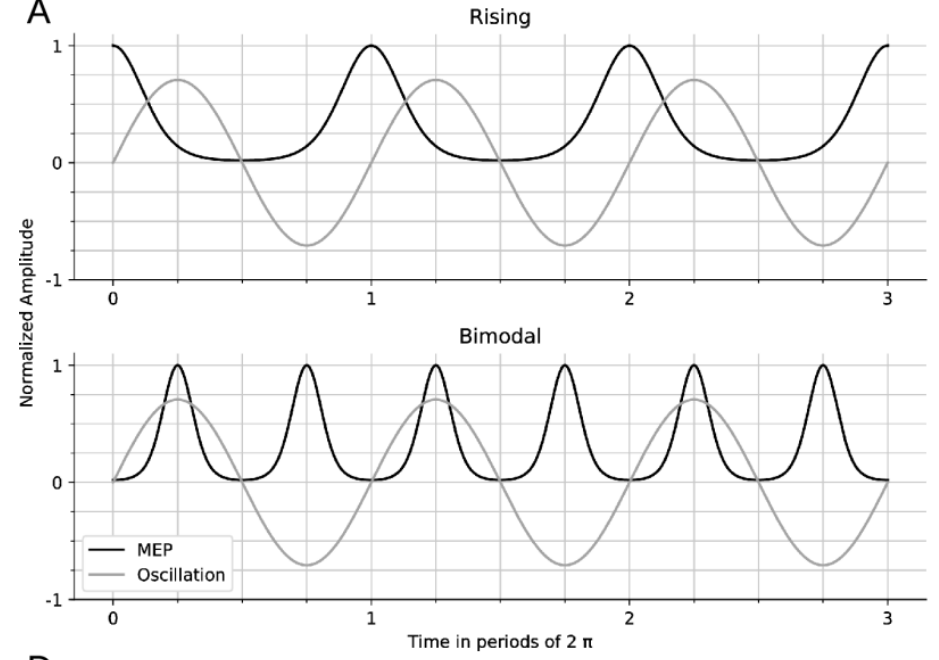

$\mathrm{D}$
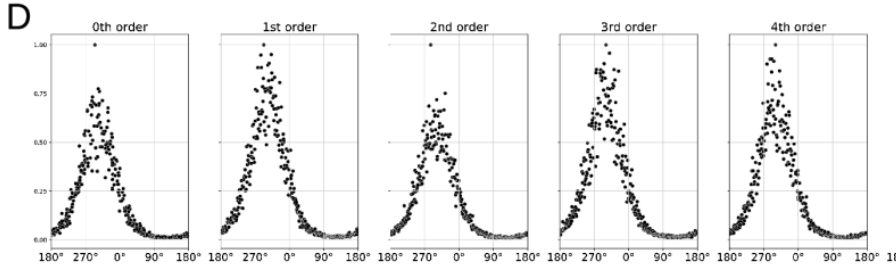

B
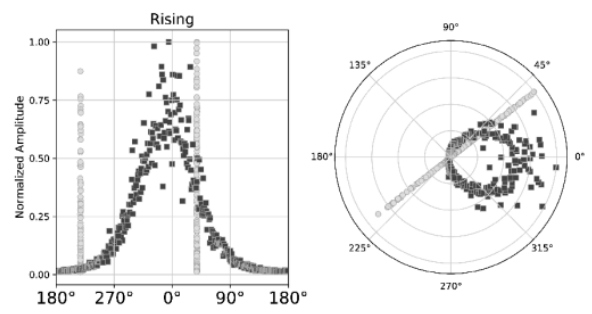

C
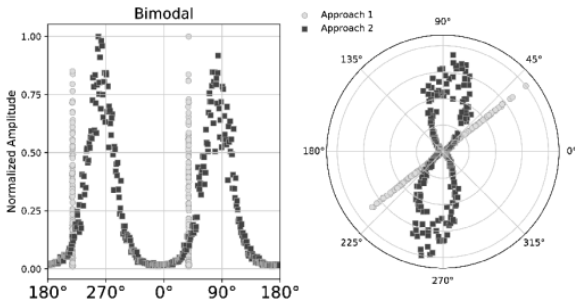

$\mathrm{E}$
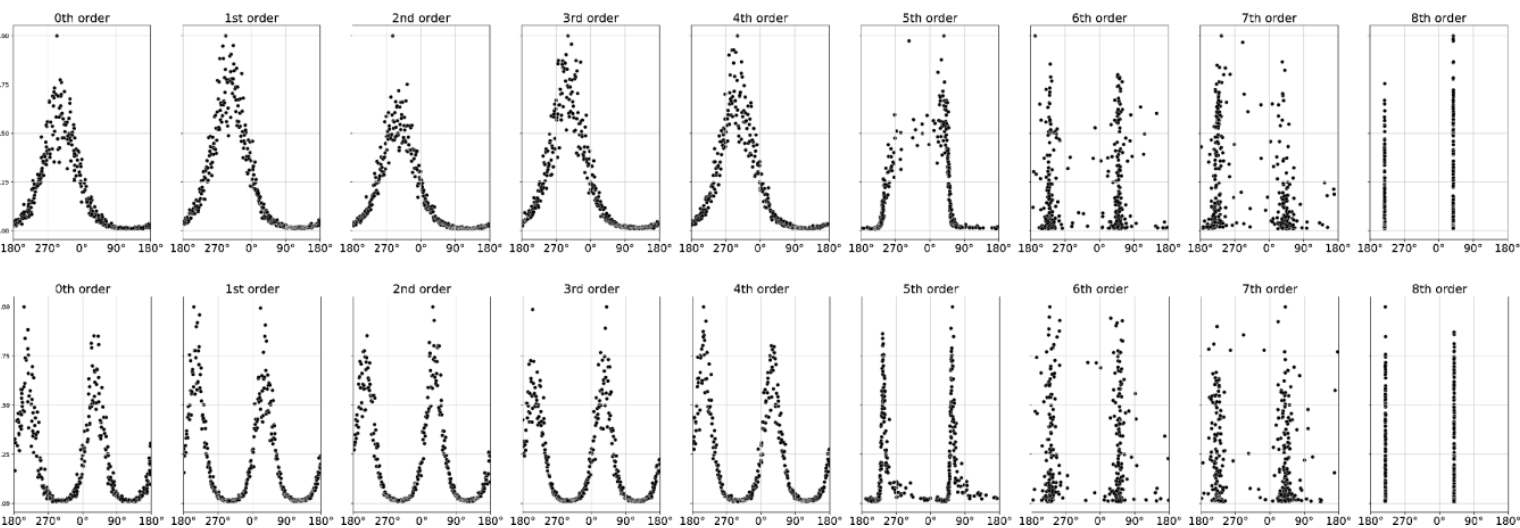

\section{Figure 1 Recovery of uni- and bimodal models depending on the approach 210 applied}

$211 \boldsymbol{A}$, Upper row shows unimodal phase-dependency with maximum MEP amplitude

212 (black line) at the rising flank of oscillatory activity (gray line). Lower row shows

213 bimodal phase-dependency with maximum MEP amplitude (black line) at the peak

214 and trough of oscillatory activity (gray line). B Unimodal phase-dependency which is

215 not recovered by narrow-band filtering followed by Hilbert transformation (approach 1,

216 light dots) but by broad-band filtering followed by discrete Fourier transformation

217 (approach 2, dark dots).C Bimodal phase-dependency which is recovered by

218 approach 2 (dark dots) and in a distorted way by approach 1 (light dots). Left columns

219 are scatter plots with phase on the $\mathrm{x}$-axis and recovered MEP amplitude on the $y$-axis.

220 Right columns show the same data as a polar plot on the unit circle. D/E The recovery

221 depending on filter order for approach 1, i.e., narrow-band $(17-19 \mathrm{~Hz})$ filtering followed

222 by Hilbert transformation with different filter orders (from zero to $8^{\text {th }}$ ) for the unimodal

223 (E) or the bimodal (F) model. Lower filter orders can distinguish between bimodal and

224 unimodal dependencies of MEP amplitudes. Note that, due to the estimation of the

225 phase 5 ms prior to the TMS-pulse, a phase-shift occurs even without filtering. 


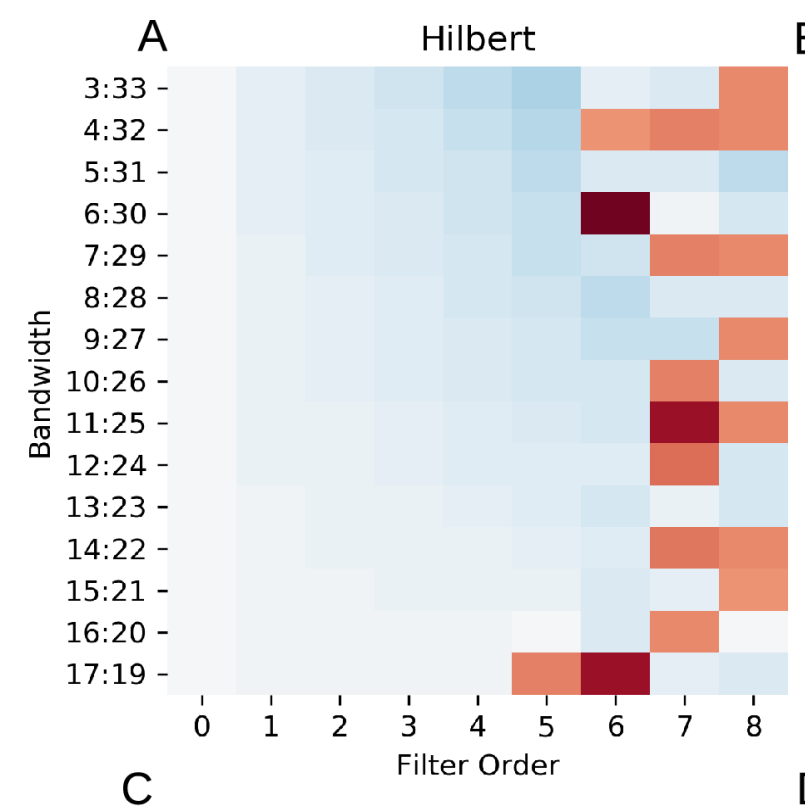

B Fourier

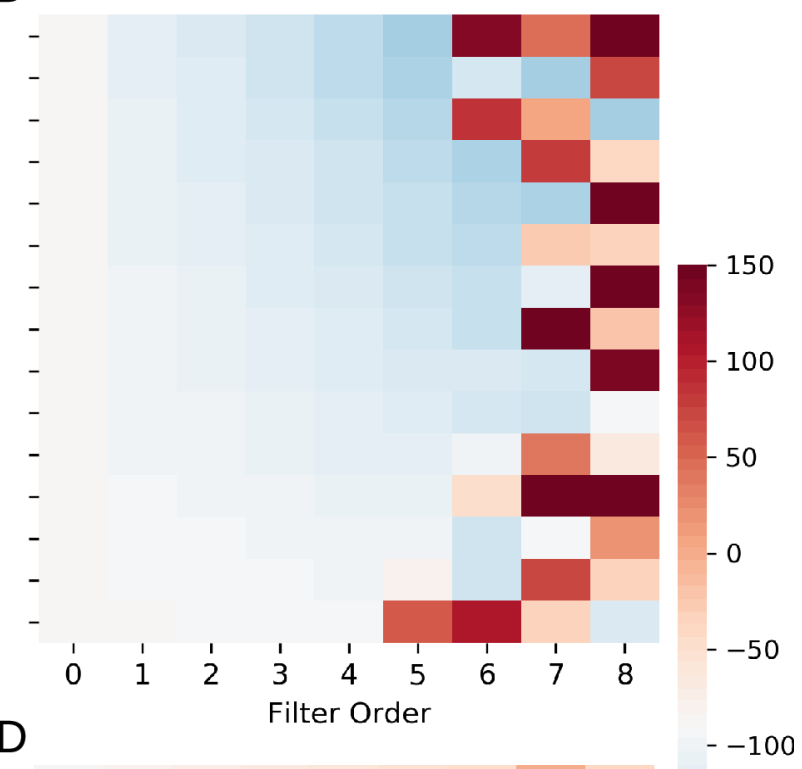

10:40 -

11:39 -

12:38 -

12:36 -

12:34 -

둥 $13: 33$

₹ 14:30

鬲 $14: 28-$

15:27 -

16:26 -

$16: 24$ -

$16: 22$ -

$17: 21$ -

18:20 -
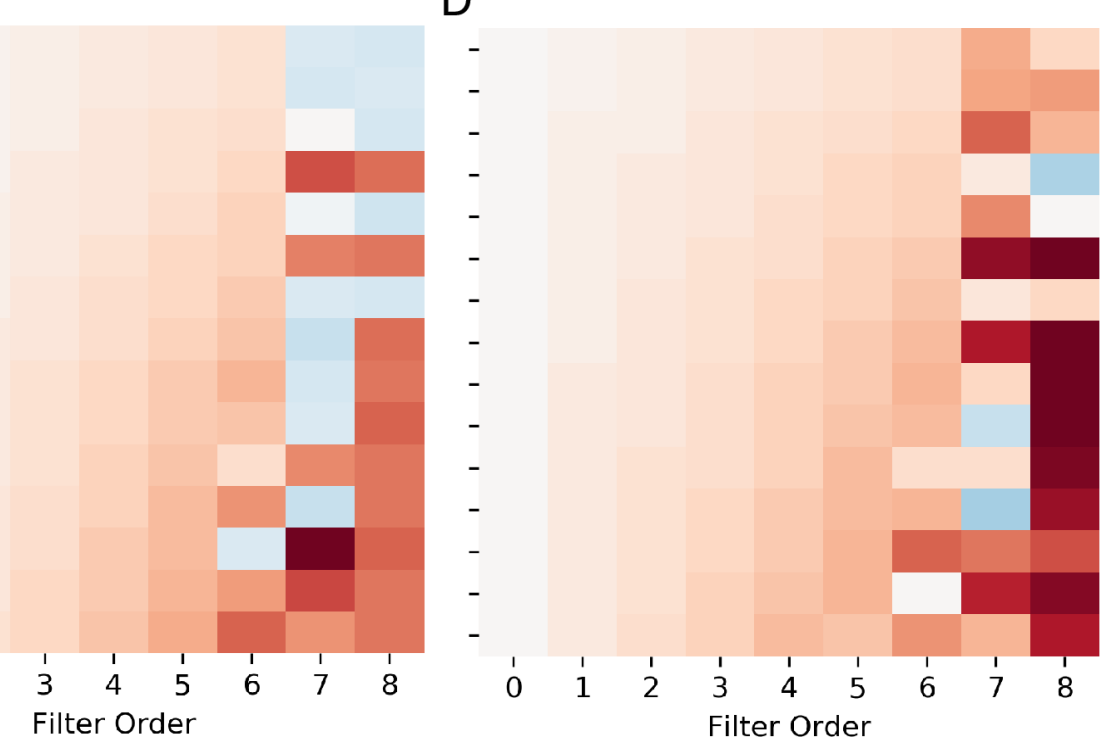

226 Figure 2 Estimated optimal phase for the unimodal dependency depends on 227 filter width, center and order

228 All heat maps show, with filter order on the x-axis and bandwidth on the $y$-axis, the 229 estimated optimal phase color-coded with a divergent colormap. The colormap is 230 anchored with white to -90 , i.e., the optimal phase according to the simulation. The 231 rows show the recovery depending on whether Hilbert $(A / C)$ or Fourier $(B / D)$

232 transformation was used for phase estimation. The columns show the recovery for 233 evenly centered $(A / B)$ or uneven bands (C/D). 